\title{
PROCESOS DE AGENTIVIDAD Y NUEVOS REPERTORIOS TRANSLINGÜÍSTICOS DE LOS ESTUDIANTES DE ORIGEN MAGREBÍ EN GALICIA ${ }^{(1)}$
}

\author{
Adil MOUSTAOUI SRHIR* \\ Universidad Complutense de Madrid \\ Gabriela PREGo VÁZQUEZ* \\ Universidade de Santiago de Compostela \\ Luz ZAS VARELA* \\ Universidade de Santiago de Compostela
}

BIBLID [1133-8571] 26 (2019) 07.1-18.

Resumen: Este artículo analiza cómo los procesos de agentividad son cruciales en la gestión del nuevo repertorio translingüístico de los estudiantes de secundaria de origen marroquí y argelino en Galicia. Los datos que analizaremos se componen de un corpus multimodal que incluye: interacciones orales y escritas, un chat de WhatsApp y prácticas comunicativas diversas, recogidas en el marco de una investigación-acción en el aula basada en un enfoque sociolingüístico etnográfico Los resultados del análisis muestran que el repertorio translingüístico emergente presenta un fuerte arraigo del árabe marroquí como lengua familiar y la incorporación de las lenguas locales -el español y el gallego- y el uso de prácticas multimodales translingüísticas, conectadas con las trayectorias transnacionales y el proceso de escolarización local de estos jóvenes.

* E-mail: adil.moustaoui@pdi.ucm.es

* E-mail: gabriela.prego@usc.es

* E-mail: luz.zas@usc.es

(1) Los resultados que se presentan en este artículo son fruto de una investigación llevada a cabo en el marco del Proyecto I+D+I «Superdiversidad lingüística en áreas peri-urbanas. Análisis escalar de procesos sociolingüísticos y desarrollo de la conciencia metalingüística en aulas multilingües». Referencia: FFI2016-76425-P. FEDER / Ministerio de Ciencia, Innovación y Universidades - Agencia Estatal de Investigación. 
Palabras clave: Prácticas translingüísticas multimodales, Multilingüismo, Identidades, Estudiantes adolescentes de origen magrebí, Galicia.

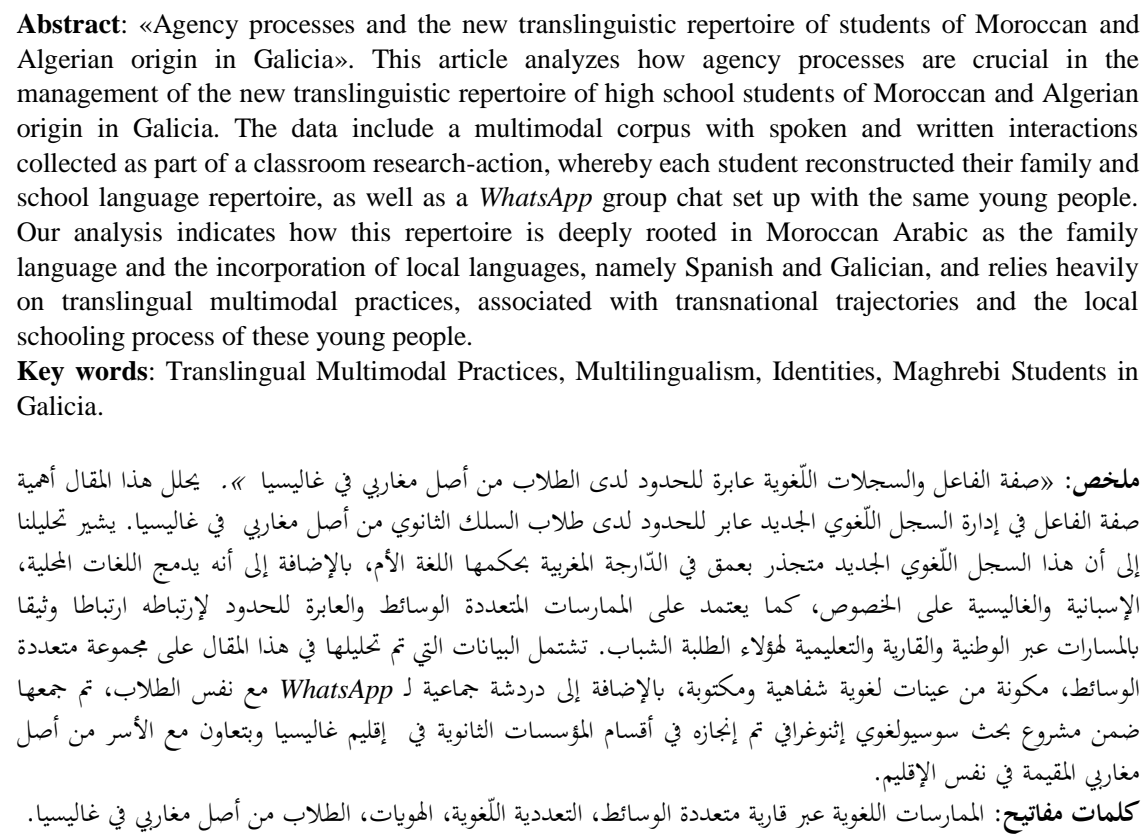

\section{Introducción}

Esta investigación, enmarcada en la Etnografía Sociolingüística Crítica, se centra en la comunidad de práctica (Wenger 2001) que conforman los estudiantes de secundaria de origen magrebí en el contexto educativo multilingüe y multicultural que actualmente caracteriza a Arteixo, municipio de A Coruña (Galicia). Así, aunque este municipio es oficialmente una comunidad bilingüe español / galego $^{(2)}$, en las últimas décadas, se ha transformado en una nueva ecología multilingüe y multicultural conectada con la migración al invertirse los flujos migratorios tradicionales del ayuntamiento (Moustaoui,

(2) El español y el gallego son lenguas cooficiales en Galicia. 
Prego \& Zas 2019; Prego Vázquez \& Zas Varela 2015; Prego Vázquez \& Zas Varela 2018; Prego \& Zas 2019; Zas y Prego 2018). Arteixo ha pasado de ser un punto de partida de emigrantes a ser un lugar de llegada. Actualmente, según los datos del Instituto Galego de Estadística (IGE) de 2018, la población asciende a 31.917 habitantes de los cuales el 5\% es población de origen extranjero ${ }^{(3)}$.

El municipio constituye una nueva ecología ruurbana multilingüe, situada en los denominados márgenes de la super-diversidad (Vertovec 2007; Mutsaers \& Swanenber 2012; Wang et al. 2014) en la que conviven diferentes lenguas y variedades: variedades lingüísticas del gallego con otras del español (español de Galicia, Venezuela, Uruguay, Argentina o Colombia), árabe marroquí, árabe argelino, wolof, ruso, rumano o portugués, hasanía, entre otras. Estas lenguas y variedades configuran marcas simbólicas de auto-identificación y de autoafirmación (Bourdieu 1982) de las diferentes nacionalidades y comunidades alóctonas que conviven en el municipio entre las que destaca cuantitativamente el grupo de origen magrebí: los estudiantes de secundaria de origen magrebí (marroquíes mayoritariamente) suponen en torno a un $0.8 \%$ del total del alumnado frente a las otras nacionalidades de migrantes.

Sin embargo, hemos puesto el foco en esta comunidad, no sólo por su relevancia cuantitativa en el municipio y en el contexto educativo, sino también, porque consideramos que los jóvenes de origen magrebí conforman una comunidad con más visibilidad social que las otras comunidades alóctonas y que, además, constituyen una comunidad de práctica en el contexto educativo en la que sus miembros establecen una red de relaciones sociales densas y múltiples (Milroy 1980), con un fuerte liderazgo de los jóvenes de origen marroquí. Además de ser los estudiantes de origen marroquí más numerosos que los argelinos, éstos últimos adoptan también la variedad de árabe marroquí de la región de Tadla-Azilal -situada en el centro sur de Marruecos-, para sus interacciones conversacionales en el grupo, tal como hemos observado en la recogida etnográfica que llevamos realizando desde el año 2016 en el ámbito educativo de este ayuntamiento.

En este marco, el objetivo de este trabajo es describir y analizar el nuevo repertorio translingüístico de los estudiantes adolescentes de origen magrebí y

(3) Hay que tener en cuenta que en este porcentaje no se incluyen las personas que obtuvieron la nacionalidad española, los extranjeros no censados y, en el caso de los adolescentes, aquellos que, aun siendo de origen alóctono, nacieron en Galicia. 
explicar en qué medida su emergencia está conectada con diferentes procesos de agentividad. Concretamente, se pretende responder a las siguientes preguntas de investigación a partir del análisis de un corpus de datos multi-situado:

- ¿Qué lenguas y variedades conforman el repertorio translingüístico de esta comunidad de práctica?

- ¿Qué procesos de agentividad tienen relevancia en la gestión del nuevo repertorio translingüístico multilingüe?

- ¿En qué medida estos procesos repercuten en las diferentes formas de mantenimiento y resistencias lingüísticas?

\section{Enfoque metodológico: marco multidisciplinar y corpus multimodal}

El corpus se complementa con diferentes tipos de datos recogidos en el marco de una investigación-acción realizada en el aula que ha incluido: (1) Grupos de discusión, mediante la técnica del puzzle de Jigsaw (Aronson 1978), en las que se reconstruyen biografías lingüísticas; (2) Actividad de «el árbol de las lenguas» con el objetivo de visibilizar y compartir las lenguas familiares; (3) Entrevistas longitudinales; (4) Un grupo de discusión a través de un chat de WhatsApp (Dud@sxlingu@s) en el que los participantes reflexionaron sobre las diferentes lenguas de su trayectoria familiar; (5) Encuesta sociolingüística cuantitativa (Rodríguez Neira, Prego Vázquez, Zas Varela, en prensa).

Como resultado, obtenemos un corpus multimodal que incluye, por un lado, interacciones orales y escritas que fueron recogidas en el aula en el marco de actividades de conciencia lingüística crítica para reflexionar sobre el repertorio lingüístico familiar y escolar; $\mathrm{y}$, por otro lado, prácticas comunicativas en un chat de WhatsApp que iniciamos con los mismos jóvenes. La recogida etnográfica de datos es multi-situada y multimodal con la finalidad de elaborar un corpus que englobe datos de distintos contextos. La finalidad es reconstruir la perspectiva émica de los actores sociales ante estas nuevas realidades multilingües. En definitiva, este corpus multi-situado nos permite contrastar, en primer lugar, las prácticas meta-comunicativas en contextos formales e informales y observar, en segundo lugar, las divergencias entre la conciencia metalingüística y las prácticas efectivamente realizadas.

La investigación se desarrolla también en un marco multidisciplinar que aúna la Etnografía Sociolinguística Crítica (Fairclough 1990, 1999; Heller 2002), la Sociolingüística Interaccional (Gumperz 1982) y la aproximación didáctica denominada Critical Language Awareness (García 2008; Hawkins 
1984, 1992). Metodológicamente, se utiliza el análisis de marcos (Goffman, 1981) y se introduce los conceptos de translanguaging (García 2009) y translanguaging moments (Wei 2018) para abordar las prácticas híbridas y la emergencia de este nuevo repertorio translingüístico multilingüe (García et al. 2018). En este marco metodológico, también serán relevantes para abordar la interpretación de los resultados conceptos como agencia o habitus, procedentes de las teorías bourdieanas (Bourdieu 1977).

\section{Resultados del análisis:}

3.1. ¿Qué lenguas y variedades conforman el repertorio translingüístico de esta comunidad de práctica?

Según los datos obtenidos en la encuesta sociolingüística que hemos realizado en el curso académico 2018-2019 ${ }^{(4)}$ (Rodríguez Neira, Prego Vázquez \& Zas Varela, en prensa), 78 estudiantes entre 12 y 17 años, declaran tener conocimientos de árabe, de los cuales 52 expresan tener un nivel entre alto y medio de árabe marroquí, mientras que 26 perciben que su nivel es bajo. Con respecto al dominio del árabe estándar, 46 consideran que su nivel es alto y medio mientras que el resto manifiesta que su nivel es bajo. Sin embargo, el conocimiento de otras variedades de árabe y de tamazight es menor: 18 encuestados dicen tener un dominio alto / medio de otras variedades de árabe -sobre todo la estándar- y 13 de tamazight. A continuación, en la siguiente imagen, se incluye la pregunta número 15 de la encuesta en la que los estudiantes deben responder acerca de sus competencias lingüísticas:

Ejemplo 1. Pregunta $n^{\circ} 15$ de la encuesta sociolingüística.

15. Podes entender a un nivel...

Marca as linguas e o nivel que tes con cada una de las lenguas. Se non as falas marca a opción "Nada". Debes marcar todas as linguas ainda que non as coñezas.

Nada

Baixo

Medio

Alto

Galego

Español/Castelán

(4) En la encuesta sociolingǘstica ha participado todo el alumnado de los centros educativos de Educación Secundaria y Bachillerato del ayuntamiento. 


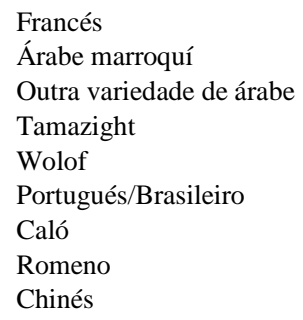

Por otro lado, el análisis cualitativo de las prácticas comunicativas obtenidas en la investigación-acción muestran que el repertorio translingüístico emergente presenta un fuerte arraigo al árabe marroquí como lengua familiar y, asimismo, observamos la incorporación de las lenguas locales -el español y el gallego-, así como el uso de prácticas multimodales translinguísticas, conectadas con las trayectorias transnacionales y el proceso de escolarización local de estos jóvenes. En los datos analizados observamos que todos los estudiantes, excepto uno, manifiestan que dariya / árabe marroquí es la lengua más importante para ellos. El dariya / árabe marroquí constituye su lengua familiar y es la más habitual en la comunidad de práctica. La imagen que aparece a continuación es un ejemplo que ilustra la reconstrucción biográfica familiar en la que dariya ocupa un lugar importante:

\section{Ejemplo 2. Árbol lingüístico de una estudiante de origen marroquí.}

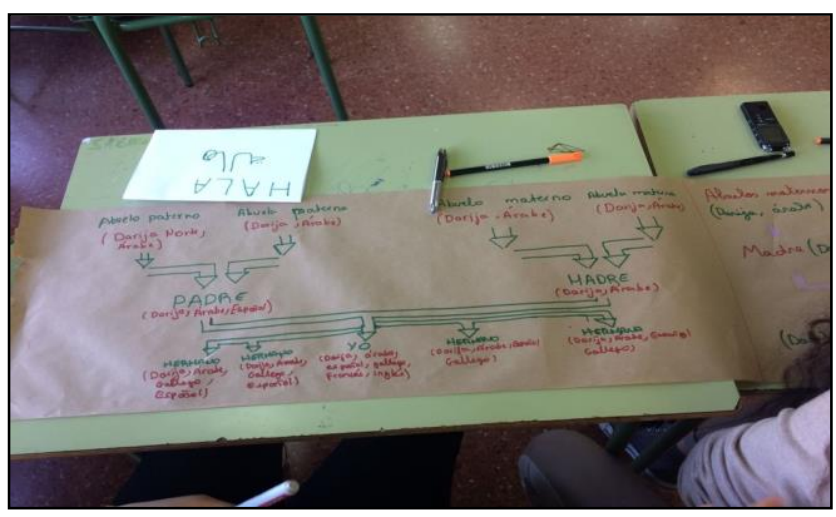


Esta lengua indexicaliza un cronotopo relevante «deslocalizado» y transnacional en el que estos jóvenes se sitúan en un espacio social desterritorializado y configurado entre Galicia y Marruecos. Así, estos participantes desde su punto de anclaje físico en «el aquí / ahora de Galicia» se orientan hacia su cultura de origen. Por lo tanto, el dariya es importante por su estatus social de lengua mayoritaria y de lingua franca en Marruecos, como consecuencia es la variedad lingüística que más usan los adolescentes en «el aquí en Galicia» con su familia y amigos. Esta situación es la mayoritaria debido a que todavía es muy poco frecuente que haya familias mixtas. El siguiente ejemplo da cuenta del repertorio de CG, el único chico que considera el español como lengua tan importante como dariya. CG procede de una familia mixta cuya madre es gallega y su padre es marroquí. Por otra parte, el gallego, la lengua propia de Galicia, no ocupa un papel relevante entre las preferencias de estos chicos.

Ejemplo 3. Multilingüismo en una familia de origen marroquí.

CG: Eh os voy a decir esto. Mi abuelo materno habla español y gallego. Mi abuela materna habla español y gallego. Mi abuela paterna habla árabe y dariya. Mi abuelo paterno habla árabe, francés y dariya. Mi madre habla español y gallego, y marroquí....ah no mucho a ver. Mi padre español, gallego, francés, dariya, chleuh y tamazight. Y yo hablo español, gallego, francés, inglés, árabe y chleuh... poco. Y mis hermanos español la mayoría, hab... árabe, francés, inglés y el gallego.

AL: no se entiende.

CG: Me da igual

El análisis de los datos nos muestra que las prácticas translingüísticas dariya / español/gallego/ francés son bastante frecuentes entre estos jóvenes e incluso reconocen que es habitual que usen entre ellos lo que denominan «el mezclado», una variedad de translanguaging español de Galicia, gallego / dariya y a veces francés como se ilustra en los siguientes ejemplos obtenidos del chat. Estos dos episodios muestran lo que podríamos considera translanguaging moments (Wei, 2018). En primer lugar, el aribizi, sistema de escritura alternativo que utiliza el alfabeto latino para representar el árabe y el dariya, es el código de escritura diferente como se refleja en estos ejemplos: 


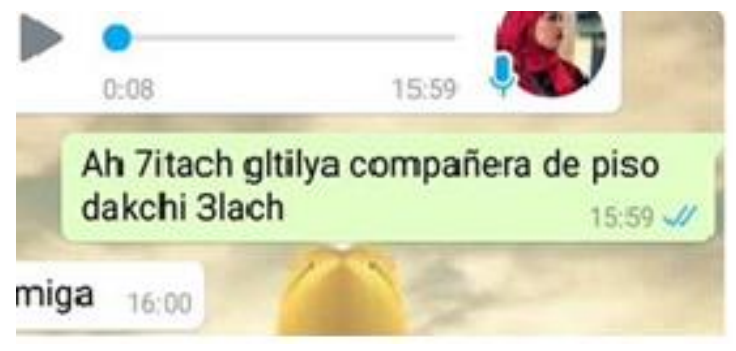

Figura 1. Ah 7itach gltilya compañera de piso dakchi 3lach (Ah porque me comentaste que fue la compañera de piso y por eso)

En segundo lugar, observamos, cómo se desdibujan las fronteras linguiísticas en la interacción: «Ah 7itach gltilya compañera de piso dakchi 3lach».

En esta misma línea, los ejemplos de las figuras 3 y 4 que vienen a continuación, revelan el papel de las prácticas híbridas en la construcción de estos espacios deslocalizados que constituyen los denominados translanguaging spaces (Wei 2011). 


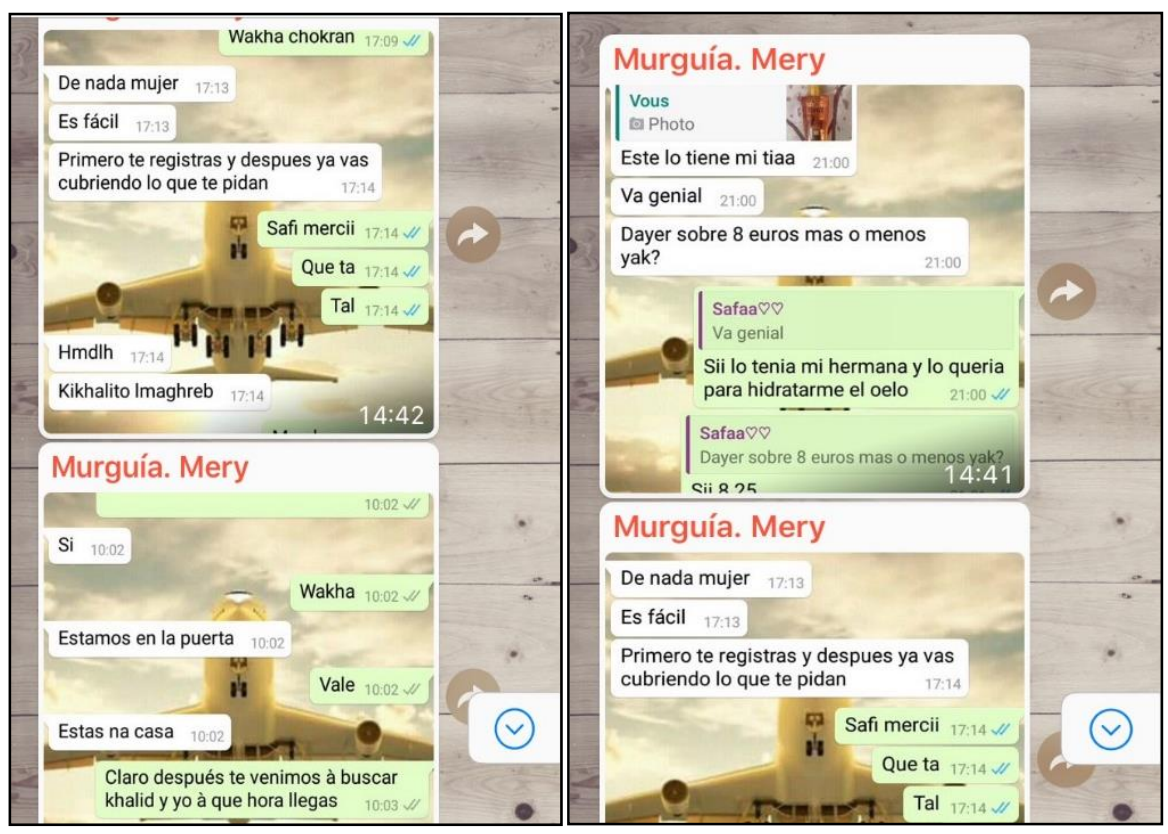

Figura 2.

Figura 3.

Vous (imagen de un producto)

Este lo tiene mi tiaa

Va genial

Dayer 8 euros mas o menos (vale 8 euros mas o menos)

Si lo tenia mi hermana y lo queria para hidratarme el oelo Sii 8.25

De nada mujer

Es fácil

Primero te registras y después ya vas cubriendo lo que pidan

Safi merci (Vale gracias)

Que ta

Tal

Hmdlh (Hamdu Allah / Gracias a Dios)

Kikhalito lmaghreb (Cómo habéis dejado Marruecos)

$\mathrm{Si}$ 
Wakha (vale)

Estamos en la puerta

Vale

Estas na casa

Claro después te venimos à buscar Khaled y yo à que hora llegas.

En definitiva, estos ejemplos (Figura 3 y 4) conectan bien con lo que señala (Wei 2011, 1223) en la media en que según el autor:

[...] translanguaging space is particularly relevant to multilinguals not only because of their capacity to use multiple linguistic resources to form and transform their own lives, but also because the space they create through their multilingual practices, or translanguaging, has its own transformative power informal. It is as space where the process of what Bhabba (1994) calls "cultural translation"' between traditions takes place; it is not a space where different identities, values and practices simply co-exist, but combine together to generate new identities, values and practices.

3.2. ¿Qué procesos de agentividad tienen relevancia en la gestión del nuevo repertorio translinguiístico multilingüe?

\subsubsection{La agentividad parental en el mantenimiento de la lengua familiar}

Tal como señalamos en anteriores investigaciones (Moustaoui 2019a; Moustaoui 2019b; Moustaoui, Prego \& Zas 2019) los procesos de agentividad, tanto parental como de los adolescentes, repercuten claramente en las diferentes formas de mantenimiento, gestión del repertorio translingüístico y resistencias lingüísticas.

El repertorio lingüístico descrito en los ejemplos anteriores es el resultado del entrecruzamiento entre la agentividad parental y la agentividad de los adolescentes en el seno de la comunidad de práctica que forman estos jóvenes. La investigación etnográfica revela tres procesos cruciales de agentividad (Bourdieu 1977) que se entrecruzan para formar un habitus (Bourdieu 1977) transnacional (Guarnizo 1997). Esta agentividad parental se manifiesta a través de las políticas lingüísticas destinadas al mantenimiento de las lenguas de origen. Mediante estas políticas las familias promueven actividades dirigidas al aprendizaje de lengua y cultura árabe y la transmisión de la religión en tanto que herencia, no sólo religiosa o cultural sino también como espacio para aprender y reproducir las prácticas lingüísticas y culturales de sus países de origen. En este sentido, ambos centros culturales de la comunidad magrebí en el municipio de 
Arteixo imparten actividades religiosas y de enseñanza de árabe clásico a la que acuden la mayor parte de niños y jóvenes.

Por otro lado, la socialización linguiística de los jóvenes de origen magrebí se realiza mayoritariamente en las variedades lingüísticas de origen en el contexto familiar. En sus hogares se siguen las cadenas televisivas marroquíes o del mundo árabe y mantienen, a través de las redes sociales, una relación continuada con familiares y amigos de su lugar de origen al que regresan con sus padres casi todos los veranos.

Las familias promueven también otras actividades dirigidas al aprendizaje de la lengua y cultura árabe como las que ofrece el Programa de Lingua árabe e cultura marroquí (PLACM). Este programa es el resultado de un convenio de cooperación cultural entre el gobierno de España y de Marruecos cuyo objetivo principal es enseñar la lengua árabe y la cultura marroquí al alumnado marroquí escolarizado en centros educativos de enseñanza primaria y secundaria, si bien el programa también permite al alumnado y profesorado no marroquí la participación en estas clases. En el curso 2018-2019 estaban matriculados 47 estudiantes del centro educativo; la mayor parte eran estudiantes de origen marroquí y también acudían estudiantes argelinos. No participa ningún alumno ni docente autóctono o de otra nacionalidad. Casi la totalidad de los estudiantes marroquíes y argelinos del centro acuden a esta actividad.

En este sentido, la agentividad parental cobra una especial relevancia en la vitalidad lingüística de las lenguas de origen en los jóvenes de origen magrebí y en garantizar una cohesión sociolingüística en el seno de las familias y las comunidades lingüísticas a las que pertenecen (Moustaoui 2019a).

En definitiva, la familia constituye un espacio de desarrollo del habitus transnacional, promovido por la agentividad parental, y que se forja en un proceso de construcción de un espacio deslocalizado en el que se conecta el allá (Marruecos) y el aquí (Galicia) a través de una serie de prácticas sociales, lingüísticas, religiosas, económicas y políticas (Giddens 1991; Guarnizo 1994; Ostergaard Nielsen 2003; Vertovec 2007). Los factores religiosos, de herencia cultural y socio-comunicativos, mediados por la agentividad parental, hacen que emerjan ideologías positivas asociadas al árabe y especialmente al dariya por ser lengua materna. Dichas ideologías favorecen, por lo tanto, el proceso de capitalización de las lenguas de origen en la diáspora y propician la transmisión y mantenimiento de estas lenguas en el seno de las familias. 


\subsubsection{La agentividad de los adolescentes en la gestión del multilingüismo familiar}

Como hemos podido observar en el ejemplo 1,2 y 3 , tanto en la actividad de «el árbol de las lenguas» como en «la encuesta sociolingüística», las diferentes variedades del gallego y del español también forman parte del repertorio comunicativo de estos jóvenes. Su socialización en el contexto escolar y en otros espacios diferentes a su entorno familiar es fundamental en la adquisición de estas dos lenguas. El contexto educativo cobra especial importancia porque es un lugar de encuentro continuado entre los grupos autóctonos y alóctonos. En este espacio desterritorializado de aprendizaje y de contacto entre diferentes comunidades de prácticas y de hablas, los adolescentes adquieren un nuevo repertorio multilingüe propio que incorporan a un espacio familiar a través de sus prácticas lingüísticas. Como resultado, estos jóvenes marroquíes incorporan este nuevo repertorio multilingüe a su espacio familiar a través de sus prácticas lingüísticas. La agentividad de los adolescentes es ahora fundamental para articular el perfil multilingüe de estas familias. Así, el análisis de los datos nos muestra que las prácticas translingüísticas dariya / español / gallego / francés son bastante habituales entre estos jóvenes e incluso reconocen que es habitual que usen entre ellos. Constituye, pues, una variedad «mezclado» de translenguar que desafía las normas monolingües de la escuela, las instituciones y también las fronteras políticas, geográficas, territoriales y lingüísticas de la comunidad o del Estado-nación en el que residen.

\section{3. ¿En qué medida estos procesos repercuten en las diferentes formas de mantenimiento y resistencias lingüísticas?}

Tal como hemos confirmado en investigaciones anteriores (Moustaoui, Prego \& Zas 2019), el uso de las diferentes redes sociales adquiere un lugar muy destacado en las prácticas interaccionales de los jóvenes. Según el Estudio General de Medios (abril 2018 - marzo 2019) los adolescentes utilizan la mensajería instantánea como un medio habitual de comunicación, fundamentalmente WhatsApp. Las conversaciones en soporte digital se incorporan de forma creciente a su red de relaciones y, por lo tanto, se han convertido en un agente de socialización muy relevante en el mundo actual. A través de las tecnologías se han ido modificando hábitos de relación y con independencia del lugar en el que vivan los chicos y chicas mantienen 
relaciones en redes deslocalizadas y conectadas por internet. Estas peculiaridades son de suma importancia en el caso de adolescentes que mantienen una red de relaciones en sus lugares de origen y como comprobaremos, permiten entender y abordar nuevas formas de resistencia en las que la creatividad multimodal tiene un papel muy relevante.

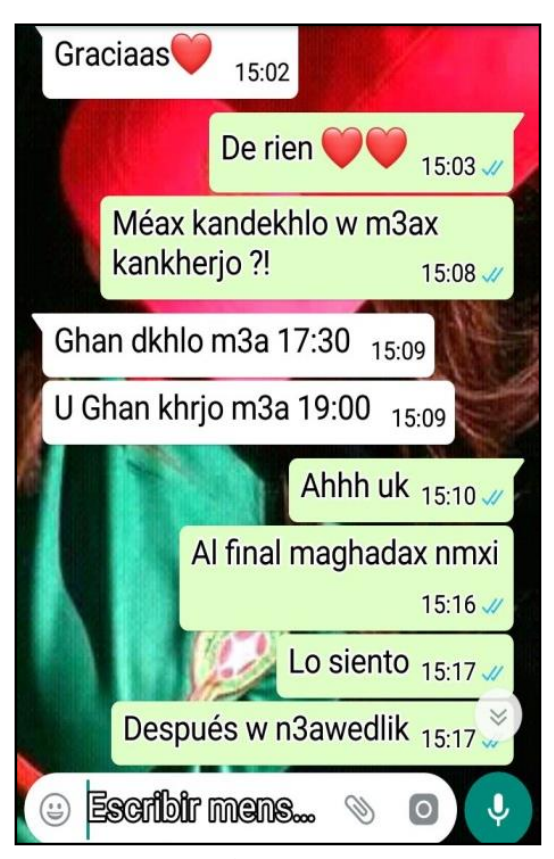

Figura 5.

(Traducción Figura 5.)

Gracias

De nada

¿A qué hora entramos y a qué hora salimos?

Entramos a las 17:30

Salimos a las 19:00

Ah. Ok.

Al final no voy a ir

Lo siento

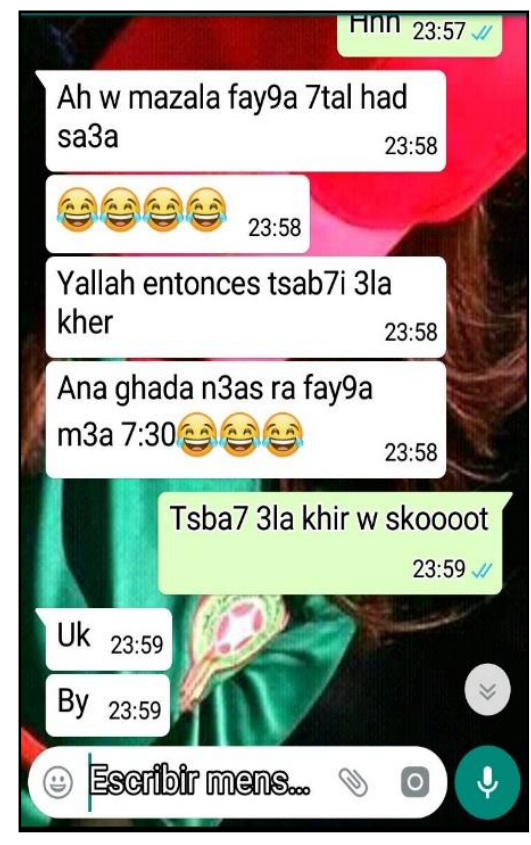

Figura 6.

(Traducción Figura 6.)

AH Sigues despierta a estas horas.

Que tengas entonces una buena noche

Me voy a dormir que mañana me despierto a las 7:30

Que tengas buenas noches Y No hables más.

Ok

by 
Después te contaré

Estas prácticas comunicativas espontáneas multilingües son formas de creatividad y resistencia lingüística al uso del árabe estándar y del español. Constituyen acciones con un especial valor indéxico con las que se gestionan los denominados translanguaging spaces (Wei 2011) en los que se forja la identidad «ser joven marroquí en la diáspora».

\section{Conclusiones}

Los datos que hemos analizado en este artículo muestran que el repertorio translingüístico de los estudiantes de origen magrebí, se configura como un continuum en el que las fronteras entre las lenguas se desdibujan en los translanguaging moments (Wei 2018), espacios indéxicos de las nuevas identidades y valores de «ser jóvenes de origen magrebí en la diáspora». Los jóvenes de origen magrebí constituyen, por tanto, una comunidad de práctica visible en el contexto educativo y generan nuevo conocimiento a través de experiencias compartidas en las que, por un lado, aprenden y crean cooperativamente nuevas prácticas comunicativas híbridas como el arabizi y como «el mezclado». Así, por un lado, hemos visto que el arabizi, sistema de escritura no estandarizado que consiste en escribir el árabe o dariya con alfabeto latino, está totalmente extendido en sus prácticas comunicativas digitales. Y, por otro lado, el «mezclado», una práctica de translanguaging español / gallego / dariya (a veces el francés), muestra la capacidad creativa de estos jóvenes y constituye un importante recurso de resistencia lingüística.

Hemos visto, del mismo modo, cómo estos jóvenes mantienen un fuerte arraigo al árabe marroquí y al árabe estándar. Los factores culturales, sociocomunicativos y religiosos, así como la agentividad y la ideología parental juegan un papel importante en cómo se asocia no sólo el arraigo, sino también la herencia con las dos variedades del árabe, la marroquí y la estándar. Con lo cual, la capitalización de las lenguas de origen en la diáspora propicia las condiciones favorables para la transmisión y el mantenimiento de estas lenguas en el seno de las familias. Por tanto, quienes dejasen de hablar la lengua renegarían de su identidad, se asimilarían y, consecuentemente, perderían su herencia lingüística y cultural. Y, como se ha señalado anteriormente, la escolarización local es importante en este proceso y clave en la incorporación en sus interacciones cotidianas de las lenguas oficiales del país de acogida: el español y el gallego, en menor medida. 
El resultado del análisis y de nuestro trabajo etnográfico nos ha permitido detectar el papel de diferentes tipos de agencia (Bourdieu 1977) como procesos que subyacen a la formación de este repertorio multilingüe. Como se ha explicado en el apartado 3, la multi-agency es el resultado del entrecruzamiento entre la agentividad parental y la agentividad de los adolescentes. Concretamente, se han observado tres procesos cruciales de agentividad que se entrecruzan para formar un habitus transnacional: (a) la agentividad parental manifestada en las políticas lingüísticas y educativas de transmisión y mantenimiento de la lengua de origen; (b) la agentividad de los adolescentes en la gestión del multilingüismo familiar y de sus repertorios lingüísticos; (c) la agentividad de los adolescentes en manifestar y visibilizar nuevas formas de resistencia lingüística en el discurso digital.

Finalmente, debemos señalar que tanto el papel de este nuevo repertorio comunicativo en la gestión identitaria y como la movilización de prácticas multimodales translingüísticas (Moustaoui 2016; Pereiro, Pego y Zas 2017), sirve para reconstruir las identidades de ser joven magrebí en la diáspora y es, a través de ellas, con las que se negocian las tensiones que se producen debido a las distintas formas de pertenencia que manifiestan estos adolescentes.

\section{BIBLIOGRAFÍA}

ARONSON, Elliot. 1978. The jigsaw classroom. CA, Beverly Hills.

AIMC. 2019. Audiencia de Internet en el EGM. Abril 2018/marzo 2019, España, Asociación para la Investigación en Medios de Comunicación. <https://www.aimc.es/egm/audiencia-internet-egm/> [19 de abril de 2019].

ALCÁNTARA PLA, Manuel. 2014. «Las unidades discursivas en los mensajes instantáneos de wasap». Estudios de Lingüística del Español 35, pp. 223242.

ALLEHAIBY, Wid H. 2013. «Arabizi: An Analysis of the Romanization of the Arabic Script from a Sociolinguistic Perspective». Arab World English Journal 4, 3, pp. 52-62.

BAKHTIN, Mijail. 1989. Las formas del tiempo y del cronotopo en la novela. Ensayos sobre Poética Histórica. Madrid, Taurus. 
BOURDIEU, Pierre. 1977. Outline of a Theory of Practice. Cambridge, Cambridge University Press.

FAIRCLOUGH, Normand. 1990. Critical Language Awareness. London, Longman.

FAIRCLOUGH, Normand. 1999. «Global capitalism and critical awareness of language». Language Awareness 8, 2, pp. 71-83.

GARCÍA, Ofelia. 2008. «Multilingual language awareness and teacher education». In: Jasone, Cenoz \& Nancy, Hornberger. Encyclopedia of Language and Education. Vol 6. Berlin, Springer, pp. 385-400.

GARCÍA, Ofelia. 2009. «Education, multilingualism and translanguaging in the 21st century». In: Ajit, Mohanty Minati Panda, Robert, Phillipson \& Tove, Skutnabb-Kangas. Multilingual Education for Social Justice: Globalising the local. New Delhi, Orient Blackswan (former Orient Longman), pp. 128-145.

GOFFMAN, Erving. 1981. Forms of Talk. Oxford, Blackwell.

GIDDENS, Anthony. 1991. Modernity and Self Identity: Self and Identity in the Late Modern Age. Cambridge, Cambridge University Press.

GUARNIZO, Luis Eduardo. 1997. «The Emergence of a Transnational Social Formation and the Mirage of Return Migration among Dominican Transmigrants». Identities 4, 2, pp. 281-322.

GUMPERZ, John. 1982. Discourse Strategies. Cambridge, Cambridge University Press.

HAWKINS, Eric. 1984. Awareness of language: An introduction. Cambridge, Cambridge University Press.

HAWKINS, Eric. 1992. «Awareness of language / knowledge about language in the curriculum of England and Wales. An historical note on twenty years of curricular debate». Language Awareness 1, 1, pp. 5-17.

HELLER, Monica. 2002. Éléments d'une sociolinguistique critique. Paris, Didier.

LERNER, Gene. 1996. «On the Place of Linguistic Resources in the Organization of Talk-in-Interaction: 'Second Person' Reference in MultiParty Conversation». Pragmatics 6, 3, pp. 281-294.

MANCERA, Ana. 2016. «Usos lingüísticos alejados del español normativo como seña de identidad en las redes sociales». Bulletin of Spanish Studies, 93, pp. 1469-1493. 
MANCERA, Ana y Ana PANO. 2013. El español coloquial en las redes sociales. Madrid, Arco Libros.

MARTÍN ROJO, Luisa. 2016. «La conversación guasap». Soprag 4, 1, pp.108134.

MARTÍN ROJO, Luisa. 2010. Constructing inequality in multilingual classrooms. Berlin, Mouton de Gruyter.

MILROY, Lesley. 1980. Language and social networks. Oxford, Blackwell.

MOUSTAOUI SRHIR, Adil. 2016. «Tú serás el responsable ante Dios el día del juicio si no le enseñas árabe (a tu hijo-a): lengua árabe, identidad y vitalidad etnolingüística en un grupo de marroquíes en Madrid». Lengua y Migración 8, 1, pp. 51-79.

MOUSTAOUI SRHIR, Adil. 2019a. «Política lingüística en familias transnacionales de origen marroquí: Ideologías, prácticas y desafíos». In: Mònica Barrieras y Carla Ferrerós (eds.). Transmissions. Estudis sobre la transmissió lingüística. Eumo, Vic, pp. 179-210.

MOUSTAOUI SRHIR, Adil. 2019b. «Making Children Multilingual: Language Policy and Parental agency in Transnational and Multilingual Moroccan Families in Spain». Journal of Multilingual and Multicultural Development 40, pp. 5- 21.

MOUSTAOUI SRHIR, Adil, Gabriela PREGO VÁZQUEZ \& Luz ZAS VARELA. 2019. «Translingual Practices and Reconstruction of Identities in Maghrebi Students in Galicia». Languages 4, 3, pp. 2-16

MUTSAERS, Paul \& SWANENBER, Jos. 2012. «Super-diversity in the margins? Youth language in North Brabant, The Netherlands». Sociolinguistic Studies 6, 1, pp. 65-89.

OSTERGAARD NIELSEN, Eva. 2003. «The Politics of Migrants: Transnational Political Practices». International Migration Review 37, 3, pp. 760-786.

PEREIRO, Carme, PREGO VÁZQUEZ, Gabriela \& ZAS VARELA, Luz. 2017. «Traxectorias lingüísticas de mulleres marroquíes en Galicia». In: Laura Rodríguez Salgado \& Iria Vázquez Silva (eds.). Muller inmigrante, Lingua e sociedade. Vigo, Galaxia, pp. 189-212.

PREGO VÁZQUEZ, Gabriela \& ZAS VARELA, Luz. 2015. «Identidades en los márgenes de la superdiversidad: prácticas comunicativas y escalas sociolingüísticas en los nuevos espacios educativos multilingües en Galicia». Discurso y Sociedad 9, 1/2, pp. 165-196. 
PREGO VÁZQUEZ, Gabriela \& ZAS VARELA, Luz. 2019. «Unvoicing practices and the scaling (de)legitimization process of linguistic 'mudes' in classroom interaction in Galicia (Spain)». Journal of the International Sociology of Language 257, pp. 77-107.

RODRÍGUEZ Neira, PREGO VÁZQUEZ, Gabriela y ZAS VARELA, Luz (en prensa). «Encuesta sociolingüística para las nuevas realidades multilingües en los centros educativos: Datos cuantitativos y cualitativos cara a cara».

RAMPTON, Ben. 1995. «Language crossing and the problematisation of ethnicity and socialization». Pragmatics 5, 4, pp. 485-513.

SILVERSTEIN, Michael. 1976. «Shifters, Linguistic Categories and Cultural descriptions». In: Basso Keithh \& Selby Henry A. (eds.). Meaning in Anthropology. Alburquerque, University of New Mexico, pp. 11-56.

VÁZQUEZ-CANO, Esteban, MENGUAL-ANDRÉS, Santiago \& ROIG-VILA, Rosabel .2015. «Análisis lexicométrico de la especificidad de la escritura digital del adolescente en WhatsApp». Revista de Lingüística Teórica y Aplicada 53, 1, pp. 83-105.

VERTOVEC, Steven. 2007. «Super-diversity and its implications». Ethnic and Racial Studies 30, pp. 1024-1054.

WEI, Li. 2011. «Moment Analysis and translanguaging space: Discursive construction of identities by multilingual Chinese youth in Britain». Journal of Pragmatics, 43, 5, pp. 1222-1235.

WEI, Li. 2018. «Translanguagins as a Practical Theory of Language». Applied Linguistics 39, 1, pp. 9-30.

WENGER, Etienne. 2001. Comunidades de práctica: aprendizaje, significado e identidad. Barcelona, Paidós. 